Son muy pocas las publicaciones realizadas hasta ahora sobre la hepatotoxicidad de los IECA. Fue descrita por primera vez con el captopril (8), y posteriormente también con el enalapril (5) y el lisinopril(9). En 1993, Hagley y colaboradores publicaron una revisión crítica y exhaustiva de todos los casos publicados al respecto hasta ese momento (19 casos en 17 pacientes) (2). Todos los pacientes recogidos estaban recibiendo al mismo tiempo otras medicaciones potencialmente hepatotóxicas, lo que no sucedía en nuestro caso. Dado el empeoramiento progresivo del patrón colostásico y la ausencia de datos al respecto en la bibliografía, decidimos instaurar empíricamente el tratamiento con corticoides. Nuestra impresión es que podría existir una relación temporal entre su administración y la mejoría de los parámetros hepáticos. No obstante, la variabilidad en la evolución descrita en los escasos precedentes publicados, no permite descartar que se tratase de una mera coincidencia .

E. Fernández Alons, J. M. San Román Montero, N. Cabello Clotet, P. Gargantilla Madera, A. Garzarán, J. Vergas García, A. Pardo Redondo

Servicio de Medicina Interna IV. Hospital Clínico San Carlos. Madrid

1. Aparte de los 19 casos de hepatotoxicidad en relación con el captopril recogidos por Hagley y colaboradores en su revisión publicada en 1993 , sólo hemos encontrado otro publicado en 1996 en la búsqueda que realizamos a través del MEDLINE.

2. Hagley MT, Hulisz DT, Burns CM. The Annals of farmacotherapy 1993 Febrero, vol 27: 228-231.

3. Bellary SV, Issacs PET, Scott AWM. Captopril and the liver (letter). Lancet 1989; 2: 514

4. Zimmerman HJ. Drug-induced liver disease. Drugs 1978; 16: 25-45.

5. Shionori H, Nomura S, Oda H, Kimura K, Takasaki I, Takagi N et al. Hepatitis associated with captopril and enalapril but not with delapril in a patient with congestive heart failure receiving chronic haemodialysis. Current Therapy Res. 1987; 42: 1171-1176.

6. Hagley MT, Benak RL, Hulisz DT. Suspected cross-reactivity of enalapril- and captopril-induced hepatotoxicity. Ann pharmacother. 1992; 26: 780-781.

7. Kaminsky DL. Arachidonic acid metabolites in hepatobiliary physiology and disease. Gastroenterology 1989; 97: 781-92.

8. Vandenburg M, Parfrey P, Wright P, Lazda E. Hepatitis associated with captopril treatment. Br. J. Clin. Pharmacol. 1981; 11: 105-106.

9. Larrey D, Babany G, Bernauau J, Andrieux J, Degoff C, Pessayre D. Fulminant hepatitis after lisinopril administration. Gastroenterology 1990; 99: 1832-3.

\section{Infección diseminada por Mycobacterium scrofulaceum en un paciente infectado por el VIH}

\section{Sr. Director:}

Las micobacterias no tuberculosas (MNT) y mayoritariamente el grupo Mycobacterium avium-intracellulare, son una de las principales causas de infección diseminada en pacientes infectados por el virus de la inmunodeficiencia humana (VIH) $(1,2)$. Dentro del grupo de las MNT, se encuentra el Mycobacterium scrofulaceum, del que sólo se han descrito en la literatura 6 casos de infección diseminada por dicha micobacteria en personas portadoras del VIH (Tabla I). Creemos oportuno describir un nuevo caso con características diferenciales.

Varón de 38 años, ex-adicto a drogas por vía intravenosa, con serología positiva para VIH desde hace 6 años. En febrero98, ingresa en nuestro centro, por datos clínicos sugestivos de infección respiratoria, con patrón intersticial en la radiografía de tórax; los hemocultivos, y análisis de esputo (cultivo, BAAR y Lowenstein), fueron negativos; recibió tratamiento con TMPSMX, rifampicina, isoniazida, y pirazinamida a dosis habituales, con buena evolución clínica, siendo enviado a su domicilio, después de un mes. Posteriormente al alta hospitalaria, se informa del crecimiento de una MNT en un hemocultivo. No acudió a las revisiones programadas. Vuelve a ingresar en agosto-98, por síndrome constitucional de un mes de evolución, con adelgazamiento de $6 \mathrm{kgs}$, tos, expectoración purulenta, febrícula, disnea progresiva, y disfagia en los últimos 15 días. Había abandonado el tratamiento antibiótico, hacía 3 meses. Tenía temperatura de $38^{\circ} \mathrm{C}$, tensión arterial 90/60 mm de $\mathrm{Hg}, 24 \mathrm{rpm}$, $85 \mathrm{lpm}$, caquéctico, con candidiasis en oro-farínge, en la auscultación pulmonar existían roncus aislados siendo el resto de la exploración normal. Tenía hemoglobina 11,3 gr/dL, VCM 83,3 fL, leucocitos $2.900 / \mathrm{mm}^{3}$, linfocitos $200 / \mathrm{mm}^{3}$, CD4 $1 / \mathrm{mm}^{3}$ $(0,6 \%)$, plaquetas $200.000 / \mathrm{mm}^{3}$; el estudio de coagulación era normal, así como la bioquímica excepto, GOT $40 \mathrm{UI} / \mathrm{L}$, proteinas $6 \mathrm{~g} / \mathrm{dL}$, albúmina $3 \mathrm{~g} / \mathrm{dL}$, y sodio $132 \mathrm{mEq} / \mathrm{L}$. En la gasometría tenía pO2 $55 \mathrm{~mm}$. La radiografía de tórax presentaba enfermedad intersticial con imágenes nodulares aisladas. En el cultivo de esputo se aisló Enterobacter cloacae, Kleibsiella pneumoniae, Aspergillus fumigatus y Candida albicans. No se detectó Pneumocystis carinii en esputo. Los hemocultivos fueron negativos. La carga viral del VIH era de 1.112.216 copias/mL. En la endoscopia digestiva presentaba úlceras esofágicas sugestivas de infección por citomegalovirus. El estudio de fondo de ojo fue normal. La búsqueda de BAAR en esputo, orina, y sangre fue negativa. Recibió tratamiento inicialmente con cefotaxima, ganciclovir y ciprofloxacino vía intravenosa, completado con itraconazol (sustituido posteriormente por anfotericina), isoniazida, rifampicina, y pirazinamida, por vía oral. A pesar del tratamiento, persistían la febrícula vespertina, la expectoración abundante, y algún episodio de hemoptisis ocasional. En noviembre-98, se identifica la MNT como Myco bacterium scrofulaceum en el hemocultivo del ingreso anterior (febrero-98), y en un Lowenstein de esputo del ingreso actual (agosto-98). El tratamiento fue modificado pasando a rifabutina, etambutol, claritromicina, D4T, Ddi, y nelfinavir; el ganciclovir fue suspendido al confirmar la cicatrización de las úlceras esofágicas; fue alta hospitalaria en diciembre-98 con una gran mejoría en la situación clínica, así como de las imágenes radiológicas; la cifra de CD4 era de $40 / \mathrm{mm}^{3}(3,6 \%)$, y la carga viral de VIH tenía 4.835 copias/ml, con $\log 3,68$.

El M. scrofulaceum es una micobacteria no tuberculosa ubicua, contaminante del agua y del suelo (3), responsable fundamentalmente de linfadenitis en niños inmunocompetentes entre 1-5 años $(4,5)$, y que puede producir infecciones diseminadas, en pacientes con algún tipo de inmunosupresión (6). En los últimos años, principalmente en España y en Estados Unidos, se han descrito de forma muy escasa, infecciones diseminadas por M. scro fulaceum en pacientes portadores del VIH, con inmunosupresión severa (cifras de CD4 $<100 / \mathrm{mm}^{3}$ ), presentándose clínicamente con fiebre, síntomas respiratorios, digestivos, lesiones cutáneas, y en ocasiones con imágenes patológicas en parénquima pulmonar y mediastino (5-8).

La evolución clínica en la mayoría de los casos registrados, fue desfavorable (la mitad de ellos murieron), condicionada por la existencia simultánea de factores desfavorables: grave deterioro clínico e inmunológico de los pacientes, presencia de otras infecciones oportunistas, demora en la identificación del M. scro fulaceum, y resistencia por parte de esta micobacteria a los fármacos antituberculosos de primera línea (9).

A diferencia de los casos publicados con anterioridad, la evolución clínica del paciente que presentamos fue satisfactoria, en principio con medicación antituberculosa habitual, con recaída tras su abandono, y posteriormente con recuperación clínica final, coincidiendo con terapéutica a base de claritromicina, rifabutina, etambutol, y tratamiento antiretroviral. 
INFECCIÓN DISEM INADA POR M YCOBACTERIUM SCRO FULACEUM EN PACIENTES INFECTADOS POR EL VIH

Caso

Clínico

Referencia

Bibliográfica

Edad/Sexo

País

Factores de riesgo

CD4/ $\mathrm{mm}^{3}$
Diagnóstico
SIDA previo

Síntomas

Radiografía

de tórax

\section{M uestas \\ positivas \\ para M}

scrofulaceum

Tratamiento

$$
\begin{gathered}
R F^{(\mathrm{d})}, \mathrm{PZ}^{(\mathrm{e})}, \\
E M \mathrm{~B}^{(\mathrm{f})}
\end{gathered}
$$

Fiebre,

tos,

diarrea.

2

3

6

$\begin{array}{ll}49 / M^{(a)} & 29 / H^{(b)} \\ \text { España } & \text { España }\end{array}$

Trasplante, Transfundido

\section{Transmisión} sexual

\section{4}

NO

Fiebre,

diarrea

disnea

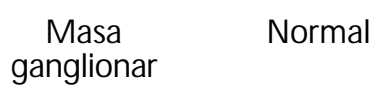

Adenopatía, Hemocultivos
muestras
respiratorias

O rina, muestras respiratorias

Fiebre, diarrea, vómitos, tos productiva astenia.

\section{nfiltrados crónicos} calcificados

\section{Estados}

Unidos

\section{Adicto a drogas} vía parenteral

\section{3}

SI

Lesión cutánea en muñeca, adelgazamiento

\section{Infiltrado}

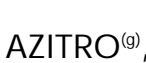
$\mathrm{RF}, \mathrm{INH}^{(\mathrm{h})}$
Biopsia

cutánea,

muestras

INH, RIF, PZ, EM B respiratorias
5

7

22/H

Bélgica

Hemofilia, transfusión

N.D. SI

Fiebre

N.D. cavitado

Biopsia de adenopatía retroperitoneal

N.D.
Caso actual

8

N.D. ${ }^{(c)}$ 38/ H España

Unidos

N.D.

Adicto a drogas vía parenteral

1

\section{SI}

Fiebre, tos productiva, disnea, adelgazamiento, disfagia

Infiltrados intersticiales y lesiones nodulares

Hemocultivo, $\begin{array}{ll}\text { uestra res- } & \text { Hemocultiva, } \\ \text { piratoria, y Esputo }\end{array}$ orina

INH, RIF, y

PIR. RIB (i),

$\operatorname{CLAR}^{(\mathrm{j})}, \mathrm{y}$ EM B

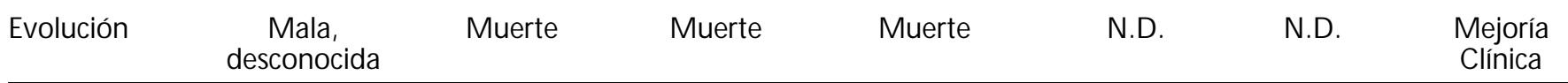

a: mujer; b: hombre; c: no documentado; d: rifampicina; e: pirazinamida; f: etambutol; g: azitromicina; $h$ : isoniazida; i: rifabutina; j: claritromicina.

Coincidimos en la opinión de otros autores, (10) al pensar que las infecciones diseminadas por M. scrofulaceum en pacientes VIH positivos, pueden ser más frecuentes de lo que las cifras apuntan, por lo que ante la sospecha clínica de dicha coinfección, se deben procesar para micobacterias, todas las muestras biológicas potencialmente contaminadas, y en especial los hemocultivos.

Quisieramos agradecer a la Unidad de Micobacterias del Servicio de Microbiología, en el Complejo Hospitalario Universitario de Santiago de Compostela, la identificación del Mycobacte rium scrofulaceum en hemocultivos y esputo.

\section{J. Núñez Fernández, R. Ojea de Castro*, L. Anibarro García**, A. Pascual Duran***}

Servicio de Medicina Interna. *Unidad de Patología Infecciosa. **Unidad de Tuberculosis. ***Servicio de Microbiología. Comple jo Hospitalario de Pontevedra. Hospital Montecelo. Pontevedra

1. Horsburg CRJr. Mycobacterium avium complex infection in the acquired immunodeficiency syndrome. N Engl J Med 1991; 324: 1332-8.

2. Herrero Martínez JA, Sánchez Manzano MD, Palenque Mataix E., Medina Iglesias P, Sánchez Rivas JL, Rubio García R, Costa Pérez-
Herrero. Infección diseminada por Mycobacterium avium-intracellulare en pacientes con SIDA. Med Clin (Barc) 1993; 100: 171-3.

3. Kirschner RAJr, Parker BC, Falkinham III JO. Epidemiology of infection by Nontuberculous Mycobacteria. Am Rev Respir Dis 1992; 145; 271-5.

4. Pang S.C. Mycobacterial lymphadenitis in western Australia. Tuber Lung Dis 1992; 76: 362-7.

5. Campos-Herrero MI, Rodríguez H, LLuch J, Perdomo M, Pérez $M^{\mathrm{a}} \mathrm{C}$ Gómez E. Infección dieseminada por Mycobacterium scrofulaceum: a propósito de 3 casos. Enferm Infecc Microbiol Clin 1996; 14: 258-60.

6. Sanders JW, Walsh AD, Snider RL, Sahn EE. Disseminated Mycobacterium scrofulaceum infection: a potentially treatable complication of AIDS. Clin Infect Dis 1995; 20: 549-56.

7. Delabie J, De Wolf-Peeters C, Bobbaers H, Bibbe G, Desmet VJ. Immunophenotypic analysis of histiocytes involved in AIDS-associated Mycobacterium scrofualceum infection: similarities with lepromatous lepra. Clin Exp Immunol 1991; 35: 214-8.

8. Shafer RW, Sierra MF. Mycobacterium xenopi, Mycobacterium fortuitum, Mycobacterium kansasii, and others nontuberculous mycobacteria in an area of endemicity for AIDS. Clin Infect Dis 1992; 15: 161-2.

9. Wolinsky E. Mycobacterial diseases other than tuberculosis. Clin Infect Dis 1992; 15: 1-12.

10. Lozano F, Nogales C, Corzo JE, Gutierrez MJ, Gómez-Mateos JM. Mycobacterium scrofuleceum y SIDA. Enferm Infecc Microbiol Clin 1996; 14: 628. 